\title{
Improved HMM for Cursive Arabic Handwriting Recognition System Using MLP Classifier
}

\author{
${ }^{1}$ Mouhcine Rabi, ${ }^{2}$ Mustapha Amrouch, ${ }^{3}$ Zouhir Mahani \\ 1,2 Laboratory IRF-SIC, faculty of sciences IbnZohr University, Agadir Morocco \\ ${ }^{3}$ High school of technology, IbnZohr University, Agadir Morocco \\ mouhcineh@gmail.com,m.amrouch@uiz.ac.ma, zouhir.mahani@uiz.ac.ma
}

\begin{abstract}
Recognizing unconstrained cursive Arabic handwritten text is a very challenging task the use of hybrid classification to take advantage of the strong modeling of Hidden Markov Models (HMM) and the large capacity of discrimination related to Multilayer Perceptron (MLP) is a very important component in recognition systems.The proposed work reports an effective method on improvement our previous work that takes into consideration the context of character by applying an embedded training based HMMs this HMM is enhanced by an Artificial neural network that are incorporated into the process of classification to estimate the emission probabilities. The experiments are done on the same benchmark IFN/ENIT database of our previous work to compare the results and show the effectiveness of hybrid classifier for enhancing the recognition rate the results are promising and encouraging.
\end{abstract}

Keywords: Arabic Handwriting Recognition; Context; Embedded training; HMMs; Multilayer Perceptron (MLP).

\section{Introduction}

Systems for handwriting recognition are referred to as off-line or on-line systems depending on whether ordinary handwriting on paper is scanned and digitized or a special stylus and a pressure-sensitive tablet are used. In both the ultimate objective is to convert handwritten sentences or phrases words or characters in analogue form (off-line or on-line sources) into digital form (ASCII).

More than 300 million people around the world speak Arabic and their derivative. Arabic is naturally written cursively in both handwritten and typewritten modes. In comparison to Latin Arabic seem to be more complex. For example many letters in this language have complementary diacritics such as dots madda and zigzag bars. In addition the letters have different shapes at different locations of the word.

Due to variability in handwriting styles and distortions caused by the digitizing process even the best handwritten word recognizer is unreliable when the number of word choices is large. This necessitates the use of advanced concepts to achieve a performance level comparable to that of humans. The researches focus on the use of new methods and approaches to perform handwriting recognition. In this area the concept of combining multiple classifiers is proposed as a new direction for the development of highly reliable handwriting recognition systems and some preliminary results have indicated that the 
combination of several complementary classifiers will improve the performance of individual classifiers [01] [02].

In current work our system performs training and recognition of words and characters. In order to model the variations related to the character context in the corpus we have opted for a specific type of learning. Therefore character models are trained and obtained through embedded training; thereafter the decision has been done by the proposed hybrid classifier which mainly based on HMMs and neural network.

The remainder of this paper is organized as follow. Section 2 presents a literature review and related works of handwriting recognition system. Section 3 is focused on our contribution starting with our developed reference system then the incorporation of hybrid classifier. The performance of the recognition system has been experimented on the benchmark database IFN/ENIT and the obtained experimental results are shown and analysed in section 4 . The paper finally closed with a conclusion and perspectives.

\section{Literature review}

Hidden Markov Models (HMMs) have proven to be one of the most successful and widely used classifiers in the area of text recognition. There are many reasons for success of HMMs in text recognition including avoidance of the need to explicitly segment the text into recognition units; characters or graphemes In addition HMMs have sound mathematical and theoretical foundation [03].

[04] The authors have investigated on contextual sub-characters HMMs for text recognition by using multi-stream HMMs where the features calculated from a sliding window frame form one stream and its derivative features are part of the second stream. The experiments were conducted with different traintest configurations on the IFN/ENIT database and the best recognition rate achieved was $85.12 \%$. In [05]Azeemused an effective technique for the recognition of offline Arabic handwritten words using Hidden Markov Models. Besides the vertical sliding window two slanted sliding windows are used to extract the features. Three different HMMs are used: one for the vertical sliding window and two for slanted windows a fusion scheme is used to combine the three HMMs. [06] proposed a combined scheme for Arabic handwritten word recognition using a HMM classifier followed by re-ranking. Basically intensity features are used to train the HMM and topological features are used for re-ranking to improve accuracy. Experiments were carried out using IFN/ENIT database and the achieved recognition rate was 83.55\%.An alternative approach is proposed in [07] and [08] where multi-stream HMM models is used this paradigm provides an interesting framework for the integration of multiple source of information. Significant experiments have been carried out on two public available database the recognition rate was $79.8 \%$ in IFN/ENIT for Arabic and 89.8\% in IRONFF for Latin script. In [08] Maqqorproposed a system of Arabic handwriting recognition based on combining methods of decision fusion approach. The combination of the multiple HMMs classifiers was applied by using the different methods of decision fusion approach. The system is evaluated using the IFN/ENIT database. Experimental results demonstrate that the Weighted Majority Voting (WMV) combination method have given better recognition rate $76.54 \%$ with Gaussian distribution. In [09] the authors present an analytical approach of an offline handwritten Arabic text recognition system. It is based on the Hidden Markov Models (HMM) Toolkit (HTK) without explicit segmentation. The feature extraction uses a sliding window on the line text image and processed by two groups of these features (the features of local densities and the statistical characteristics). The proposed system has been experimented in two different databases: "Arabic-Numbers" database where we 
Mouhcine Rabi, Mustapha Amrouch, Zouhir Mahani; Improved HMM for Cursive Arabic Handwriting Recognition System Using MLP Classifier. Transactions on Machine Learning and Artificial Intelligence, Vol 5 No 4 August (2017); pp: 20-29

achieved a rate of $80.26 \%$ for words and $37.93 \%$ for sentences and IFN/ENIT database where we achieved a rate of $78.95 \%$ for words.

Otherwise [10] proposed a new approach for the offline Arabic handwritten word recognition based on the Dynamic Hierarchical Bayesian Network (DHBN) using a free segmentation released by a smoothed vertical projection histogram with different width values. The model is consisting of three levels. The first level represents the layer of the hidden node which models the character class. The second layer models a frame set representing the sub-characters and the third layer models the observation nodes. The developed system has been experimented and the results are provided on a subset of the IFN/ENIT benchmark data base. These results show a significant improvement in the recognition rate because of the use of the DHBN. Most of the recognition errors of the proposed system can be attributed to the segmentation process error and to the poor quality of some data samples.

In many other works neural networks in its different applications have been extensively applied to classify characters as part of isolated or continuous handwritten word recognizers [11] [12] [13] [14] [15].

This paper focus on the impact of using aembedded training based on a hybrid classifier the motivation for the work on the hybrid HMMs and Artificial neural network models presented here originates from a critical analysis of the state of the art in offline handwritten text recognition [16] [17] [18] our previous work on offline handwriting recognition using HMMs [19] researches and experiences in using hybrid HMM/ANN models for automatic speech recognition [20][21][22][23] [24]and for online handwriting recognition [25]. All these criteria making hybrid modeling an important factor in order to achieve an effective and efficient system.

\section{Contribution}

\subsection{Reference System [19]}

Our reference system (figure1)was analytical without explicit segmentation based HMMs using embedded training to perform and enhance the character models.

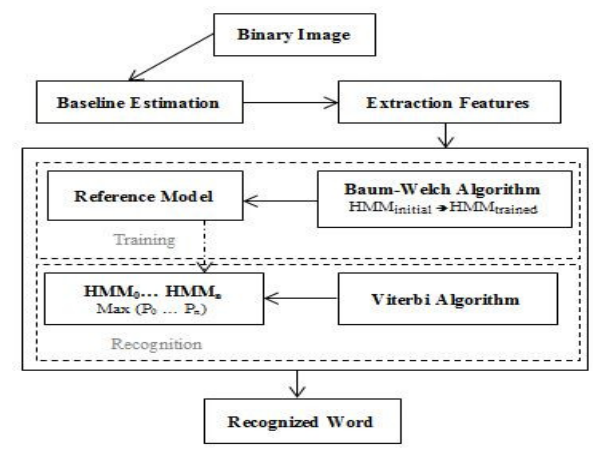

Figure.1. Synopsis of reference system

Extraction features was preceded by baseline estimation; the approach used to estimate these baseline based on the horizontal projection curve that is computed with respect to the horizontal pixel density knowing that the skew and slant correction of words are made in pre-processing step to harmonize the direction of the sliding windows in the extraction features. These latter are statistical and geometric to integrate both the peculiarities of the text and the pixel distribution characteristics in the word image. 
The sliding windows are shifted in the direction of writing (right to left). In each window we extract a set of 28 features represent the distribution features based on foreground pixels densities and concavity features. Each window is divided into a fixed number $n$ of cells. Some of these features are extracted from specific areas of the image delimited by the word baselines.

These features are modelled using hidden Markov models and trained by theembedded training method (figure 2).

We used a model for each character right-left topology with four states and three transitions for each state. Word model is built by concatenating the appropriate character models.

The embedded training is to automatically identify relevant information letters without specifying them explicitly by exploiting the redundancy of information between words matched to changes in context and letters position.

The major problem of HMMs is the estimation of emission probabilities; this confirms that HMMs are powerful to model sequences but still limited compared to NN and SVM in classification [26] for this reason and the motivations cited above in section II it is prominent and promising to use a hybrid classifier.

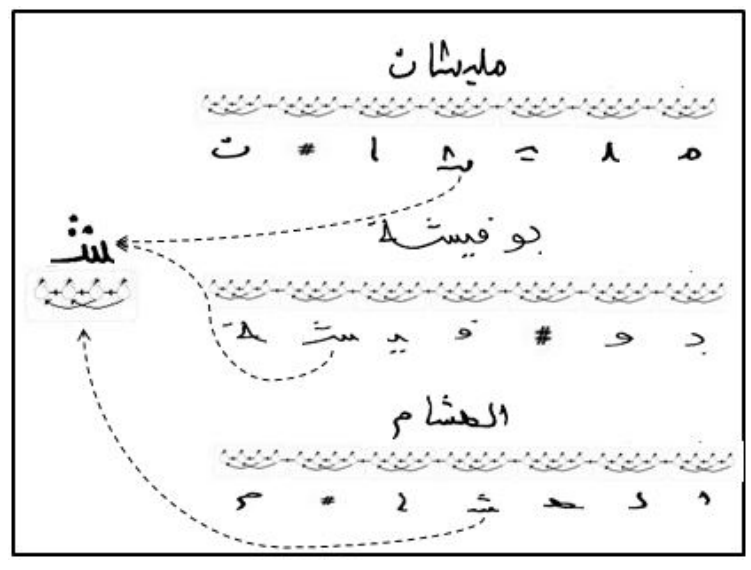

Figure.2. Embedded training of character "chin" [19]

For more explanations about the baseline system refer to [19].

\subsection{Hybrid classifier}

To improve the performance of the off-line handwritten recognition system either the accuracy ofthe classifier has to be increased. In this section we introduce the hybrid approach then we clarify the principle for our offline Arabic handwriting recognition system.

While HMMs are effective in modeling variation in handwriting they lack discrimination ability because of maximum-likelihood parameter estimation criteria. The strength of MLP is in the fact that they don't need to assume about statistical distribution of input as well as they can be trained to exhibit discriminant properties. As already mentioned recent works in various area of research tried to develop hybrid HMM/MLP systems in which MLPs are used to compute the emission probability associated with each state of HMM. 
Mouhcine Rabi, Mustapha Amrouch, Zouhir Mahani; Improved HMM for Cursive Arabic Handwriting Recognition System Using MLP Classifier. Transactions on Machine Learning and Artificial Intelligence, Vol 5 No 4 August (2017); pp: 20-29

By HMM the goal of handwriting recognition is to retrieve the most likely grapheme character or word sequence $\mathrm{W}$ given a sequence of observation vectors $\mathrm{x}$ which is achieved by maximizing the a posteriori probability:

$$
\hat{w}=\arg \max P(w \mid X)
$$

Typically each grapheme is modelled by a right to left HMM and the number of states is chosenglobally or individually for each character. Gaussianmixtures are used to model the output distributions ineach state $\mathrm{q}$ given the feature vector $\mathrm{x}(\mathrm{x} \mid \mathrm{q})$. The Baum-Welch algorithm is used for training the HMMs whereasthe Viterbi algorithm is used for recognition.

Hybrid modelsfor handwriting recognition based HMMs were built with different neural networks. [27]; [28] use an MLP. [29] built an hybrid CNN/HMM. In [30]CNNs is applied in the hybrid framework for handwritten word recognition using different segmentationmethods.

In Hybrid HMM/MLP classifier neural networks can be considered statistical classifiers under certain conditions by supplying output of a posteriori probabilities. Thus it is interesting to combine the respective capacities of the HMM and the MLP for a new efficient recognition system inspired by the two formalisms.

The principal idea behind the MLPNN/HMM hybrid approach as illustrated in Figure 3 is to estimate the output probability density function of each state of the used HMM by the output nodes of the MLP classifier which received features as input. These input vectors are pre-processed to finally estimate the posteriori probability deciding whether the input vector belongs to the desired character class. The MLPNN's output weighted by the priori probability of each class forms the probability density function used for every state of the HMM.

In the hidden Markov modeling approach the emission probability density $\mathrm{P}(\mathrm{x} \mid \mathrm{q})$ must be estimated for each state $\mathrm{q}$ of the Markov chains that is the probability of the observed feature vector $\mathrm{x}$ given the hypothesized state $q$ of the model.

In the proposed hybrid HMM/ANN approach the emission probabilities are provided with a neural network since ANNs can be trained to estimate probabilities that are related to these emission probabilities. In particular an MLP can be trained to approximate the a posteriori probabilities of states $P(q \mid x)$ if each MLP output unit is associated with a specific state of the model and if it is trained as a classifier.

The a posteriori probability estimates from the MLPoutputs $P(q \mid x)$ can be converted to emission probabilities $\mathrm{P}(\mathrm{x} \mid \mathrm{q})$ by applying Bayes rule:

$$
\mathrm{P}(x \mid \mathrm{q})=\frac{P(q \mid x) P(x)}{P(q)}
$$

The class priors $P(q)$ can be estimated from the relativefrequencies of each state from the information produced bya forced Viterbi alignment of the training data. Thus thescaled likelihoods $P(x \mid q) / P(q)$ can be used as emissionprobabilities in the proposed system since during recognitionthe scaling factor $P(x)$ is a constant for all classes. This allows MLPs to be integrated into hybrid structural connectionist models via a statistical framework. 


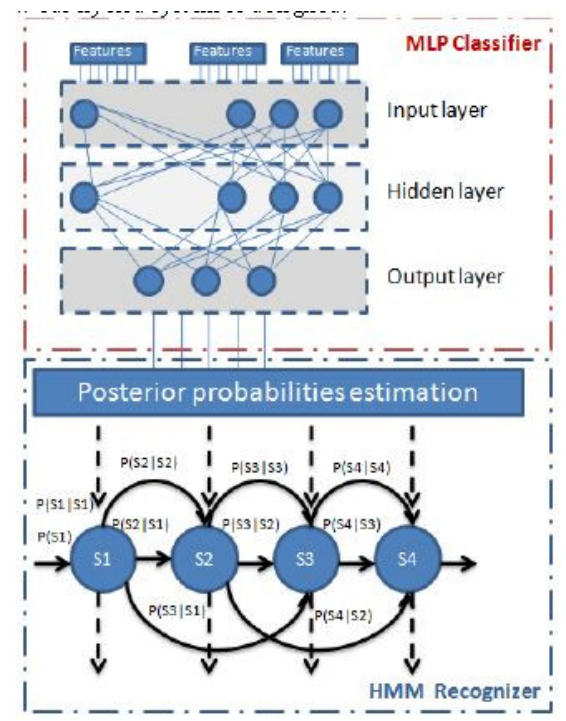

Figure 3: Global scheme design of the hybrid model HMM/MLP

The advantages of this approach are the discriminate training criterion (all MLP parameters are updated in response to every input feature vector) and the fact that it is no longer necessary to assume an a priori distribution of the data. Furthermore if left and right contexts are used at the input of the MLP important contextual information can be incorporated into the probability estimation process.Another strength of this approach is that computing emission probabilities with hybrid HMM/ANN models is usually faster than conventional HMMs with Gaussian emissions since it only requires a forward pass of the MLP for all states of the Markov chains.

\section{Experimental results}

To evaluate the performance of our recognition system experiments are conducted using IFN/ENIT [31] database of handwritten Arabic words. It was produced by the Institute for Communications Technology at the Technical University of Braunschweig (IFN) and the "EcoleNationaled'ingénieur de Tunis (ENIT) "National school of engineering Tunis."This database is used bymore than 110 research groups in about 35 countries [32].

\subsection{HMM vs HMM/MLP Classification}

Reference recognition HMM experiments were conducted using continuous density HMMs with diagonal covariance matrices of Gaussians in each state, a right-to-left topology was applied with four states for each character and three transitions for each state. The optical models were trained and tested using the HTK toolkit [33].

The developed system used hybrid classifier based HMMs enhanced by an artificial neural network that are incorporated into the process of classification to estimate the emission probabilities.

Table1 shows the experimental results of our developed system compared to reference system using the same benchmarking database IFN/ENIT to illustrate the reliability of our improvement models. 
Mouhcine Rabi, Mustapha Amrouch, Zouhir Mahani; Improved HMM for Cursive Arabic Handwriting Recognition System Using MLP Classifier. Transactions on Machine Learning and Artificial Intelligence, Vol 5 No 4 August (2017); pp: 20-29

Table1: Recognition results of improvement system compared to reference system

\begin{tabular}{ccc}
\hline System & Models & $\mathrm{RR}^{*} \%$ \\
\hline Reference & $\mathrm{HMM}$ & 87.93 \\
Hybrid & $\mathrm{HMM} / \mathrm{MLP}$ & $\mathbf{8 9 . 0 3}$ \\
\hline
\end{tabular}

RR: Recognition Rate

We compare the reference system [19] with the developed system using hybrid HMM/MLP classifier. Results in Table1 show an improvement due to embedded training using the hydride classification: accuracy is increased by $1.1 \%$.

\subsection{Comparison with other systems}

A comparison of the recognition rates of our system with other state-of-the-art systems evaluated on the IFN/ENIT database is presented.

Table2 shows the results of recognition rates for various offline systems recognition of cursive Arabic handwritten text using diverstype of models and the same database with the same configuration (trainingtesting); sets $a, b$ and $c$ for training and $d$ for test, to compare rates and infer the effectiveness of the proposed method.

Table 2: Recognition results of various systems

\begin{tabular}{ccc}
\hline System & Models & RR $^{*} \%$ \\
\hline Irfan [04] & Contextual sub character & 85.12 \\
\hline Alkhateeb[06] & HMM+ re-ranking & 83.55 \\
Kessentini[07] & Multi-stream HMM & 79.80 \\
\hline Maqqor[08] & Multiple classifier & 76.54 \\
\hline EIMoubtahij[09] & HMM & 78.95 \\
Khaoula[10] & DBN & 82.00 \\
Our system & HMM/MLP & $\mathbf{8 9 . 0 3}$ \\
\hline
\end{tabular}

As it can be noted from Table 2 most of the previous systems are based on HMM using various techniques exploiting the contextual approach, multiple HMM classifier, re-ranking or other techniques to improve HMM models and the recognition rate for the results of the systems mentioned does not exceed $86 \%$. Others used hybrid classifier such as HMM and Dynamic Bayesian Network and the recognition rate achieved was $82.00 \%$. Whereas the proposed system using HMM/MLP outperforms the results and achieve $89.03 \%$ due to enhancement of HMMs by incorporating anartificial neural network into the process of classification to estimate the emission probabilities.. This illustrates the effectiveness of embedded training to take account the context of characters to perform the models and using hybrid $\mathrm{HMM} / \mathrm{MLP}$ classifier to improve the performance of recognition system.

\section{Conclusion and perspectives}

In this paper we have enhanced the HMM based reference system by using a hybrid HMM/MLP classifier. Extracted features are statistical and geometric to integrate both the peculiarities of the text and the pixel 
distribution characteristics in the word image. These features are modelled using hidden Markov models. These models as already mentioned in [19] take into account the context of character by applying aembedded training to perform the models. In addition, the contribution in this paper is the improving of HMM modeling by incorporating MLPs to estimate emission probabilities that present the major HMM problem in order to take advantage of the strength of HMM modeling and neural networks classification. The modelling proposed has improved recognition and shown encouraging results to be perfect using annexes improvements.

Due to variability in handwriting styles even the best handwritten word recognizer is unreliable when the number of word choices is large. This forced the use of linguistic constraints to enhance HMMs modeling by a statistical language model that are incorporated as a post-processing into the process of recognition.

Statistical Language Modeling involves attempts to capture regularities of natural language in order to improve the performance of various natural language applications, e.g. , Speech recognition, Machine translation, Handwriting recognition, Information retrieval and other applications.

The goal of Statistical Language Modeling is to build a statistical language model that can estimate the distribution of natural language as accurate as possible, which could improve significantly the results especially when we extend our system for line and paragraph recognition.

\section{REFERENCES}

[1] J. H. Alkhateeb O. Pauplin J. Ren J. Jiang Performance of hidden Markov model and dynamic Bayesian network classifiers on handwritten Arabic word recognition In Knowledge-Based Systems 24 (2011) pp.680-688.

[2] Salvador Espan a-Boquera Maria Jose Castro-Bleda Jorge Gorbe-Moya and Francisco Zamora-Martinez "Improving Offline Handwritten Text Recognition with Hybrid HMM/ANN models" 2011

T.Plots and G.A.Fink; "Markov models for offline handwriting recognition: a survey" IntJ.Anal.Recongitvol 12 no 4 pp 269-298 2009.

[3] Irfan Ahmad GernotA.Fink and SabriA.Mahmoud "Improvement in Sub-character HMM Model Based Arabic Text Recognition" 2014 14th International Conference on frontiers in Handwrting Recognition.

[4] S. Azeem and H. Ahmed "Effective technique for the recognition of offline Arabic handwritten words using hidden Markov models" Int. J. Doc. Anal.Recognit.vol. 16 no. 4 pp.399-412 2013.

[5] J. AlKhateeb H. RenJinchang Jiang Jianmin Al-MuhtasebHusni Offline handwritten arabic cursive text recognition using hidden markov models and re-ranking Pattern Recognition Lett. 32 (8) 1081-1088 2011.

[6] Y. Kessentini T. Paquet A. Ben Hamadou Off-line handwritten word recognition using multistream hidden Markov models in: Pattern Recognition Letters 31 (2010) pp. 60-70

[7] A. Maqqor A. Halli K. Satori and H. Tairi Off-line recognition Handwriting combination of mutiple classifiers In 3rd International IEEE Colloquium on Information Science and Technology IEEE CIST'14 October 2014.

[8] El MoubtahijHichamHalliAkram Khalid Satori “Using features of local densities statistics and HMM toolkit 
Mouhcine Rabi, Mustapha Amrouch, Zouhir Mahani; Improved HMM for Cursive Arabic Handwriting Recognition

System Using MLP Classifier. Transactions on Machine Learning and Artificial Intelligence, Vol 5 No 4 August

(2017); pp: 20-29

(HTK) for offline Arabic handwriting text recognition" 2016

[9] KhaoulaJayech Mohamed Ali Mahjoub and Najoua Ben Amara "Arabic Handwritten Word Recognition based on Dynamic Bayesian Network" 2016.

[10] Berend-Jan van der Zwaag “Handwritten Digit Recognition: A Neural Network Demo” 2016

[11] Xu Chen Convolution Neural Networks for Chinese Handwriting Recognition 2016.

[12] Charlie Tsai “Recognizing Handwritten Japanese Characters Using Deep Convolutional Neural Networks" 2016

[13] ThéodoreBluche “Deep Neural Networks for Large Vocabulary Handwritten Text Recognition” 2015

[14] Ahmed Mahdi ObaidHazem M. El Bakry M.A. Eldosuky IVA.I.Shehab “Handwritten Text Recognition System Based on Neural Network" 2016

[15] AL-Shatnawi M. Atallah AL-SalaimehSafwan AL-Zawaideh Farah Hanna Omar Khairuddin "Offline arabic text recognition an overview" World Comput. Sci. Inform. Technol. J. 1 (5) 184-192 2011.

[16] M.T Parvez and S.A Mahmoud " Offline Arabic handwritten text recognition : A survey " ACM ComputSurvvol 45 no 2 pp 23-35 May 2013.

[17] A.Lawgali : "A Survey on Arabic Character Recognition" International Journal of Signal Processing Image Processing and Pattern Recognition. Vol. 8 No. 2 (2015) pp. 401-426.

[18] M.RabiM.AmrouchZ.MahaniD.Mammass "Recognition of cursive Arabic handwritten text using embedded training based on HMMs" Published in: Engineering \& MIS (ICEMIS) International Conference on Sept. 2016 INSPEC Accession Number: 16467172 DOI: 10.1109/ICEMIS.2016.7745330 Publisher: IEEE

[19] Mohamed ETT AOUIL Mohamed LAZAAR Zakariae EN-NAIMANI "A hybrid ANN/HMM models for arabic speech recognition using optimal codebook" 8th International Conference on Intelligent Systems: Theories and Applications (SITA), IEEE 2013.

[20] Supriya S. Surwade "Speech Recognition Using HMM/ANN Hybrid Model" International Journal on Recent and Innovation Trends in Computing and Communication ISSN: 2321-8169 Volume: 3 Issue: 64154 4157.2015

[21] A. I. G-Moral, U. S-Urena, C. P-Moreno and F. D-Maria, 2011, "Data balancing for efficient training of hybrid ANN/HMM automatic speech recognition, " IEEE trans. on audio, speech and lang. proc., Vol 19, No. 3, 468-481.

[22] Anuj Mohamed K.N. Ramachandran Nair "HMM/ANN hybrid model for continuous Malayalam speech recognition" Published by Elsevier Ltd. Selection and/or peer-review under responsibility of ICCTSD 2012 (International Conference on Communication Technology and System Design).

[23] E. Trentin, M. Gori, 2003, "Robust combination of neural networks and Hidden Markov Models for speech recognition," IEEE trans. on Neural net., Vol 14 , No. 6, 1519-1531. 
Transactions on Machine Learning and Artificial Intelligence Vol 5 No 4, Aug 2017

[24] NajibaTagouguiHoucineBoubakerMonjiKherallah Adel M. ALIMI “A Hybrid NN/HMM Modeling Technique for Online Arabic Handwriting Recognition" International Journal of Computational Linguistics Research Volume 4 Nimber 3 Septembre 2013 pp 107-118.

[25] M.RabiM.AmrouchZ.MahaniD.Mammass" Evaluation of Features Extraction and Classification Techniques for Offline Handwritten Tifinagh Recognition " Global Journal of Computer Science and Technology (USA),: C Software \& Data Engineering, Volume 16 Issue 5 Version 1.0 Year 2016.

[26] Dreuw P. Doetsch P. Plahl C. Ney H. (2011). Hierarchical hybrid MLP/HMM or rather MLP features for a discriminatively trained Gaussian HMM: A comparison for offline handwriting recognition Image Processing (ICIP) 18th IEEE International Conference on p. 3541-3544 Sept.

[27] S Espana-Boquera MJ Castro-Bleda J Gorbe-Moya F Zamora-Martinez "Improving offline handwritten text recognition with hybrid HMM/ANN models" IEEE transactions on pattern analysis and machine intelligence 33 (4) 767-779

[28] Q.GuoF.WangJ.LeiD.TuG.Li “Convolutional feature learning and Hybrid CNN-HMM for scene number recognition" Published in journal Neucomputing archive Volume 184 Issue C April 2016 Pages 78-90 Elsevier Science Publishers B.V Amsterdam The Netherlands.

[29] Bluche T. Ney H. \&kermorvant C. (2013b).Tandem HMM with convolutional neural network for handwritten word recognition.In 17th International Conference on Acoustics Speech and Signal Processing (ICASSP) (pp. 2390--2394).IEEE.

[30] M. Pechwitz, S.S. Maddouri, V. Maergner, N. Ellouze, H. Amiri IFN/ENIT - Database of Handwritten Arabic WordsCIFED 2002, Hammamet, Tunisia (2002), pp. 129-136

[31] Märgner V. El Abed H.: ICDAR 2011 - Arabic handwriting recognition competition. In: Int'I Conf. Document Analysis and Recognition pp. 1444-1448 (2011)

[32] S. Young al. the HTK Book V3.4 Cambridge University Press Cambridge UK 2006 